\title{
A Formal Definition of Culture
}

\author{
Aliaksandr Birukou, Enrico Blanzieri, Paolo Giorgini, and Fausto Giunchiglia \\ DISI, University of Trento, via Sommarive 14, Povo(TN), Italy 38100 \\ \{birukou, blanzieri,pgiorgio, fausto\}@disi.unitn.it,
}

\begin{abstract}
Globalization makes culture no more bound to a geographical area, race or religion. Multi-national companies, software developers, scientists need to take into account cultural differences when delivering products to people. The first step in dealing with culture consists in defining and representing culture of the targeted community. AI literature addressed issues of sociality, collaboration, and coordination in agent societies, but did not target the problem of defining and representing culture of a community. In this paper, we propose a formal definition of culture of a set of agents. It generalizes existing definitions of culture and it is operational in the sense that it can be applied for characterization and comparison of culture(s) existing in various communities.
\end{abstract}

\section{Introduction}

The advent of Web 2.0 lead to an explosive growth in the number of applications targeted at communities, e.g. applications supporting social navigation, collaborative editing, bookmarking and tagging. In such applications, culture is no more bound to a geographical area or a religion, as it is usually studied in anthropology. It becomes more appropriate to speak about the culture of online communities and such communities in general can not be characterized in terms of race, religion, or country. Rephrasing Axelrod [1], electronic communications allows us to develop patterns of interaction that are chosen rather than imposed by geography. Specific applications such as search engines or e-bookshops and the ways of using them become part of the culture of people. For instance, using Norton Commander file manager or preparing documents in the MS DOS 6.0 operating system, nowadays would be considered unusual to the same extent as lighting one's house with torches. Moreover, in some scenarios we can speak about societies of pure artificial agents, such as web services or programs and their specific culture, e.g. the standards implemented or the set of functionalities used. Human traders and trading agents operating on the same markets together use the same rules and develop common practices which can be referred to as culture. All this shows that grasping and representing culture becomes an important problem in computer science. Applications should be developed consistently with the culture of the target community and the notion of culture would provide support for building such applications.

AI literature on agents addresses the issues related to sociality, such as social action [2], social co-ordination architectures and social interaction strategies for 
decentralized co-ordination in multi-agent systems [3], social laws and conventions in multi-agent environments $[4,5]$, and social roles [6]. However, the issue of sociality alone does not help neither to understand what differentiates one set of agents from another nor to grasp what are the specificities of the behavior of agents of a specific society. Although in two different agent societies agents can be able to communicate with each other and perform other social actions, these two societies can be very different from each other. We claim that the concept of culture can be used to describe and compare sets of agents. Some research approaches use the notion of culture in the context of agents, see e.g. $[1,7,8]$, other provide a model for the comparison of cultures [9]. However, none of the previous research works provides a formal definition of culture that could be readily adopted for building applications for communities and applied for the characterization and the comparison of culture.

In this paper we provide a formal definition of culture. Our goal is not to provide a formalism or a reasoning framework per se, but, rather, to give an operational definition of culture that can be used for characterizing, describing, and comparing culture in different scenarios. In particular, we address the problems of development of applications according to the community culture and of characterizing culture of existing communities. We present and formalize a definition of the notion of culture of a set of agents. We define culture as a set of traits that are shared by the set and were transmitted, where traits are "characteristics of human societies that are potentially transmitted by non-genetic means" [10]. The sharing dimension is required for going from the set of personal traits of an individual to the culture of the set of agents, and to filter out such traits as divine services, marriage habits, birth rate, which only pertain to the set of agents as a whole, but not to individuals. The transmission dimension is a way of spreading culture.

The paper has the following structure: Section 2 reviews the use of concept of culture in the literature, Section 3 presents a formal definition of culture. Section 4 discusses related work and limitations of the approach, and Section 5 concludes the paper.

\section{Defining culture}

Culture is a slippery and ubiquitous concept. Initially, culture was associated with the notion of civilization tout-court. At the end of the 30s Margaret Mead put in contrast "culture" with "a culture". "Culture means the whole complex of traditional behavior which has been developed by the human race and is successively learned by each generation"([11] cited in [12]). However, specificity of the notion of culture with respect to a given human society was needed in order to study other societies. So the same citation goes on as: "A culture is less precise. It can mean the forms of traditional behavior which are characteristic of a given society, or of a group of societies, or of a certain race, or of certain area, or of a certain period of time" (cited in [12]). As a consequence, in the anthropological literature culture has been introduced as the concept denoting 
the object of study of cultural anthropology. Other definitions were proposed and they largely vary. However, they seem to converge to the notion that culture is learned [1], it is associated with groups of people and its content includes a wide range of phenomena including norms, values, shared meanings, and patterned ways of behaving [13-18]. In anthropological literature the usefulness of the notion of culture as a scientific tool has been attacked giving rise to the so-called "writing against culture movement" (see Brumann [12] for a reaction against it). The culture as defined in anthropology usually refers to societies defined in national or ethnic terms, however, the concept of culture has been recently used for describing knowledge and behavior of other groups like in the concepts of corporate culture or organizational culture $[13,19,20]$. Moreover, globalization has brought about the problem of interaction of cultures. On the one hand, such interaction leads to blurring boundaries between cultures, while on the other hand it leads to the increasing need of cultural-aware managers and professionals. Recent anthropology textbook definitions take into account the shift in meaning as, for example, in the definition by Peoples and Bailey:

Culture is the socially transmitted knowledge and behavior shared by some group of people (Peoples and Bailey [21, p. 23] cited in [12]).

Earlier authors define culture in the following ways (cited in Brumann [12]):

- Culture ... refers ... to learned, accumulated experience. A culture ... refers to those socially transmitted patterns for behavior characteristic of a particular social group (Keesing [22, p. 68]).

- Culture, or civilization, ... is that complex whole which includes knowledge, belief, art, law, morals, custom, and any other capabilities and habits acquired by man as a member of society (Tylor [23, p. 1]).

- The culture of any society consists of the sum total of ideas, conditioned emotional responses, and patterns of habitual behavior which the members of that society have acquired through instruction or imitation and which they share to a greater or less degree (Linton [24]).

- A culture is the total socially acquired life-way or life-style of a group of people. It consists of the patterned, repetitive ways of thinking, feeling, and acting that are characteristic of the members of a particular society or segment of a society (Harris [25]).

As we can see, definitions agree on the fact that culture consists of something that is shared and/or learned by a group of people, but the content of the culture varies in different definitions. Similarly to Axelrod [1], we see the content of the culture as a set of traits ${ }^{1}$, which can refer to behavior, knowledge facts, ideas, beliefs, norms, etc.

\footnotetext{
${ }^{1}$ Traits are further grouped in features in Axelrod's formulation, i.e. each feature can take value from a set of specific traits.
} 


\section{A formal definition of culture}

Consistently with AI literature, we define an agent as a "[...] physical or virtual entity that can act, perceive its environment (in a partial way) and communicate with others, is autonomous and has skills to achieve its goals and tendencies [...]" [26]. An agent can represent an individual or a collective entity such as an organization, and can have different cultural traits, which are characteristics of human societies that are potentially transmitted by non-genetic means and can be owned by an agent. The requirement "can be owned by", which we add to the definition by Mulder [10], means that it is possible for an agent to have a cultural trait. Different kinds of behavior, beliefs, knowledge, mentioned as elements of culture previously, are just particular kinds of cultural traits in terms of our formalism.

To model changes in the set of traits of an agent and consequently, changes in culture, we use the notion of state. We assume that the world can be in different states and the set of traits of the same agent can be different in different states.

Let us consider the set of agents $A g$, the set of traits $\mathcal{T}$, and the set of states $S$. Given an agent $a \in A g$ and a state $s \in S$, we denote the set of cultural traits of the agent $a$ in the state $s$ with $T_{a}(s)=\left\{\tau_{i}\right\} \subseteq \mathcal{T}$ and we use the predicate $h a s\left(a, \tau_{i}, s\right)$ to represent the fact that the agent $a$ has a trait $\tau_{i} \in T_{a}(s)$ in the state $s$. In the following, we call the set of traits of an individual the culture of an individual.

Example 1. Let us consider a set of people and model them as agents with a set of traits and a behavior related to transmission, telling_ $D A$ (telling that Dante Alighieri wrote "The Divine Comedy"). Let $A g$ in our example be a set of people: Charlie, Pedro, Maria, and Andrea are European citizens, and Toru is from Japan. Let $\mathcal{T}$ be a set of traits of different types, as shown in Table 1. For each trait, we also put its abbreviation (used in the figures later) in parentheses.

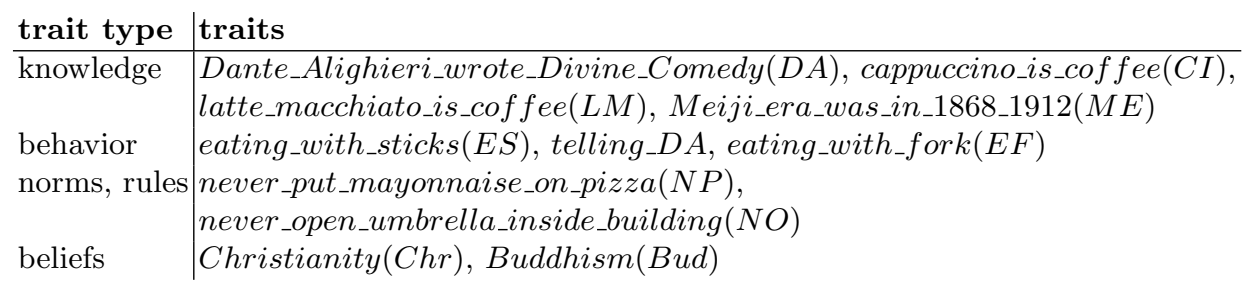

Table 1. The set of traits $\mathcal{T}$ in Example 1.

Table 2 lists the sets of traits of the specific agents of $A g=\{$ Charlie, Pedro, Toru, Maria, Andrea $\}$ in the state $s_{1}$. We can write has(Maria, Dante_Alighieri_wrote_Divine_Comedy, $\left.s_{1}\right)$, or has (Charlie, cappuccino_is_coffee, $\left.s_{1}\right)$, but not has(Andrea,eating_with_sticks, $\left.s_{1}\right)$. We will use this example as a running example. $\oslash$ 


\begin{tabular}{l|l} 
set & traits \\
\hline$T_{\text {Charlie }}\left(s_{1}\right)$ & $\begin{array}{l}\text { Dante_Alighieri_wrote_Divine_Comedy, latte_macchiato_is_coffee, } \\
\text { telling_DA, cappuccino_is_coffee, } \begin{array}{r}\text { eating_with_sticks, } \\
\text { eating_with_fork, never_put_mayonnaise_on_pizza, Buddhism }\end{array}\end{array}$ \\
\hline$T_{P e d r o}\left(s_{1}\right)$ & $\begin{array}{l}\text { Dante_Alighieri_wrote_Divine_Comedy, latte_macchiato_is_coffee, } \\
\text { cappuccino_is_coffee, eating_with_fork, Christianity }\end{array}$ \\
\hline$T_{T o r u}\left(s_{1}\right)$ & $\begin{array}{l}\text { Meiji_era_was_in_1868_1912, cappuccino_is_coffee, } \\
\text { eating_with_sticks, Buddhism }\end{array}$ \\
\hline$T_{\text {Maria }}\left(s_{1}\right)$ & $\begin{array}{l}\text { Dante_Alighieri_wrote_Divine_Comedy, latte_macchiato_is_coffee, } \\
\text { cappuccino_is_coffee, eating_with_sticks, eating_with_fork, } \\
\text { Christianity }\end{array}$ \\
\hline$T_{\text {Andrea }}\left(s_{1}\right)$ & $\begin{array}{l}\text { Dante_Alighieri_wrote_Divine_Comedy, latte_macchiato_is_coffee, } \\
\text { cappuccino_is_coffee, eating_with_fork, Christianity }\end{array}$
\end{tabular}

Table 2. Traits of agents in Example 1.

Note that we do not introduce types of traits and use them in the example only for convenience. One might propose a different classification of traits, e.g. putting eating_with_sticks as a norm. We believe that there is no single classification and it is better to deal with generic traits rather than with specific types of cultural content.

We distinguish behavior as a particular kind of traits and assume that performing a behavior by an agent changes the state of the world. In line with AI literature, we define behaviors as "[...] reified pieces of activity in which an agent engages, for example sleep or eat. In colloquial English an agent behaves in various ways; in technical AIese, an agent has various behaviors" [27]. We define the set of all behaviors $\mathcal{B} \subseteq \mathcal{T}$ and the function perform in $A g \times \mathcal{B} \times S \rightarrow S$. The intended meaning of this function is that an agent, which has some behavior in some state, performs this behavior in this state and the state of the world changes to another state. More specifically, $s_{v}=\operatorname{perform}\left(a, \tau, s_{u}\right)$ means that $h a s\left(a, \tau, s_{u}\right)$ and the agent $a$ performed a behavior $\tau$ in the state $s_{u}$ and the resulting state is $s_{v}$. The fact that $\operatorname{has}\left(a, \tau, s_{u}\right)$ does not imply that the agent $a$ is able to perform the behavior $\tau$ in the state $s_{u}$, because some preconditions for performing the behavior may be not fulfilled in the state $s_{u}$. Note that since traits are not innate, by assuming $\mathcal{B} \subseteq \mathcal{T}$ we do not include innate behaviors, such as blinking when air is puffed in someone's eye.

At this point we would like to discuss the distinction between action and behavior. In AI literature, an action is an atomic piece of activity, while behavior is perceived as something more complex, and can include several actions. Therefore, our notion of performing a behavior can really be decomposed into performing several actions. However, we decided not to introduce explicit relations between actions and behaviors. Moreover, the absence of such clear dependency in AI literature suggests that these relations are hard or even impossible to formalize. Instead, we assume that behavior can represent an atomic action or a more complex activity depending on the level of modeling granularity. We 
can vary granularity of behaviors depending on the problem in hand and on the domain. For instance, in Example 1, when someone needs to know whether agents are working, it is possible to consider behaviors working and playing, or, even, working and not_working. However, if someone would like to have a closer look at leisure activities of the group, it is necessary to introduce finer granularity of the playing behavior, e.g. by considering playing_basketball and playing_chess behaviors.

We assume that the states are ordered, we define recursively the order "is before" and the corresponding predicate is_before $\left(s_{u}, s_{v}\right)$ and is_after $\left(s_{v}, s_{u}\right)$ in the following way:

Definition 1 (is_before). is_before $\left(s_{u}, s_{v}\right) \leftrightarrow \exists a \in A g, \tau \in \mathcal{B}, s \in S$ such that $s=\operatorname{perform}\left(a, \tau, s_{u}\right) \wedge\left(s=s_{v} \vee\right.$ is_before $\left.\left(s, s_{v}\right)\right)$.

Definition 2 (is_after). is_after $\left(s_{v}, s_{u}\right) \leftrightarrow i s_{-}$before $\left(s_{u}, s_{v}\right)$

We assume that in each state $s_{v}$, the previous state $s_{u}$ is uniquely defined, while the next state depends on the action an agent performs in $s_{v}$. From Definition 1 we can derive the following property:

Property 1. For all agents $a \in A g$, for all behaviors $\tau \in \mathcal{B}$ and for all states $s_{u}, s_{v} \in S$

$$
s_{v}=\operatorname{perform}\left(a, \tau, s_{u}\right) \rightarrow i \text { is_before }\left(s_{u}, s_{v}\right)
$$

Definition 3 (sharing). For each pair of agents $a_{i}, a_{j} \in A g$, for each trait $\tau \in \mathcal{T}$, and for each state $s \in S, a_{i}$ and $a_{j}$ share the trait $\tau$ in the state $s$ iff they both have such a trait in $s$ :

$$
\operatorname{has}\left(a_{i}, \tau, s\right) \wedge \operatorname{has}\left(a_{j}, \tau, s\right) \leftrightarrow \operatorname{sharing}\left(a_{i}, a_{j}, \tau, s\right) .
$$

We also assume that agents do not lose traits when the state of the world changes, as the following axiom says:

Axiom 1 For all agents $a \in A g$, traits $\tau \in \mathcal{T}$, and states $s \in S$ :

$$
h a s(a, \tau, s) \rightarrow \forall s_{v}: i s \_a f t e r\left(s_{v}, s\right) \text { has }\left(a, \tau, s_{v}\right) .
$$

Example 1 (continued). In the example, we can write sharing(Toru, Maria, eating_with_sticks, $\left.s_{1}\right)$, or sharing(Pedro, Andrea, cappuccino_is_coffee, $\left.s_{1}\right)$, etc. To avoid giving the complete list of tuples for which sharing holds, we represent them as a graph where nodes are agents and labels on each edge denote traits that are shared by the pair of agents connected by the edge, see Figure 1 for the state $s_{1} \cdot \oslash$

Let us assume that if an agent $a_{i}$ has a trait $\tau$, the trait $\tau$ can be transmitted to another agent $a_{j}$ before some state $s$ and we use the predicate $\operatorname{transmitted}\left(a_{i}\right.$, $\left.a_{j}, \tau, s\right)$ to represent this. We represent $\operatorname{transmitted}\left(a_{i}, a_{j}, \tau, s\right)$ in a graph by a directed edge from $a_{i}$ to $a_{j}$ labeled $\tau$. 


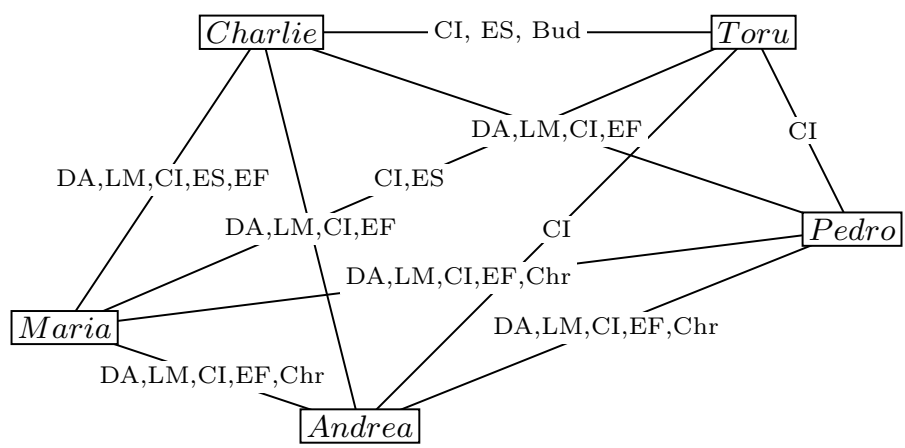

Fig. 1. The graph showing for which agents and traits the predicate sharing holds in Example 1. The nodes are agents and labels on each edge denote traits that are shared by the pair of agents connected by the edge. For instance, the edge between Toru and Andrea labeled $C I$ means that sharing(Andrea,Toru,cappuccino_is_coffee). The traits are abbreviated as in Table 1: Dante_Alighieri_wrote_Divine_Comedy is abbreviated as DA, latte_macchiato_is_coffee as $L M$, cappuccino_is_coffee as $C I$, eating_with_sticks as ES, eating_with_fork as EF, Christianity as Chr, Buddhism as Bud.

Axiom 2 For each pair of agents $a_{i}, a_{j} \in A g, a_{i} \neq a_{j}$, for each trait $\tau \in \mathcal{T}$, and for each state $s \in S$ the fact that the trait $\tau$ has been transmitted from $a_{i}$ to $a_{j}$ before the state $s$ implies that exists some state $s_{u} \in S$ such that $a_{i}$ has $\tau$ in the state $s_{u}, a_{j}$ does not have $\tau$ in the state $s_{u}$ and an agent $a_{k}$ performing $a$ behavior $\tau_{m}$ in the state $s_{u}$ imply that in the resulting state $s_{v}$ the agent $a_{j}$ has $\tau$ :

$$
\begin{gathered}
\operatorname{transmitted}\left(a_{i}, a_{j}, \tau, s\right) \rightarrow\left(\exists s_{u} \in S, \text { is_before }\left(s_{u}, s\right) \wedge \operatorname{has}\left(a_{i}, \tau, s_{u}\right) \wedge\right. \\
\left.\neg \operatorname{has}\left(a_{j}, \tau, s_{u}\right) \wedge\left(s_{v}=\operatorname{perform}\left(a_{k}, \tau, s_{u}\right)\right) \rightarrow \operatorname{has}\left(a_{j}, \tau, s_{v}\right)\right)
\end{gathered}
$$

From our assumption that traits are not innate, it follows that traits are acquired by agents, and the goal of the transmitted predicate is to show the way an agent acquired a trait. Therefore, we assume that in the initial state agents have no traits and the way they acquire traits is represented using the transmitted predicate.

We should note that the trait $\tau$ is not shared by $a_{i}$ and $a_{j}$ in the state $s_{u}$, while it is shared by $a_{i}$ and $a_{j}$ in the state $s_{v}$, and in the state $s$, as shown by the following property:

Property 2. For all pairs of agents $a_{i}, a_{j} \in A g$, for all traits $\tau \in \mathcal{T}$, and for all states $s_{v} \in S$

$$
\operatorname{sharing}\left(a_{i}, a_{j}, \tau, s_{v}\right) \rightarrow\left(\forall s: i s \_a f t e r\left(s, s_{v}\right) \operatorname{sharing}\left(a_{i}, a_{j}, \tau, s\right)\right)
$$

From Axiom 2 it also follows that the transmitted predicate holds for all subsequent states after $s_{v}$.

Property 3. For all pairs of agents $a_{i}, a_{j} \in A g$, for all traits $\tau \in \mathcal{T}$, and for all states $s_{v} \in S$ 


$$
\operatorname{transmitted}\left(a_{i}, a_{j}, \tau, s_{v}\right) \rightarrow\left(\forall s: i s_{\_} \operatorname{after}\left(s, s_{v}\right) \operatorname{transmitted}\left(a_{i}, a_{j}, \tau, s\right)\right)
$$

Example 1 (continued). Figure 2 shows the graph representing the transmitted predicate in state $s_{1}$ in our example. The traits Dante_Alighieri_wrote_Divine_Comedy and eating_with_sticks have been transmitted. On the contrary, the traits cappuccino_is_coffee and never_put_mayonnaise_on_pizza have not been transmitted (the latter trait is not even shared by any pair of agents). In particular, the Dante_Alighieri_wrote_Divine_Comedy trait has been transmitted from Charlie to Maria, and from Maria to Andrea. Also, the eating_with_sticks trait has been transmitted from Charlie to Toru and from Toru to Maria. We can write transmitted(Charlie, Maria,

Dante_Alighieri_wrote_Divine_Comedy, $\left.s_{1}\right)$.

Let us assume that in the state $s_{1}$ Charlie tells Toru that Dante Alighieri wrote the Divine Comedy and Toru memorizes this piece of knowledge. This corresponds to $s_{2}=$ perform $\left(\right.$ Charlie, telling $\left.D A, s_{1}\right)$. The transmitted predicate in the state $s_{2}$ is as depicted in the left part of Figure 2 and transmitted in the state $s_{2}$ is as depicted in the right part of Figure 2. The difference in the transmitted predicates in these two states is that the Dante_Alighieri_wrote_Divine_Comedy trait has been transmitted from Charlie to Toru and the corresponding edge is added, namely transmitted(Charlie, Toru, Dante_Alighieri_wrote_Divine_Comedy, $\left.s_{2}\right)$. In the state $s_{2}$ the following change in the set of traits for Toru occurs: $T_{\text {Toru }}\left(s_{2}\right)=\{$ Meiji_era_was_in_1868_1912, Dante_Alighieri_wrote_Divine_Comedy, cappuccino_is_coffee, eating_with_sticks, Buddhism\}.

Obviously, the transmission has an impact on sharing and the sharing predicate in the state $s_{2}$ is as depicted in Figure 3, with the edges between Toru and Charlie, Maria, Andrea, Pedro added.
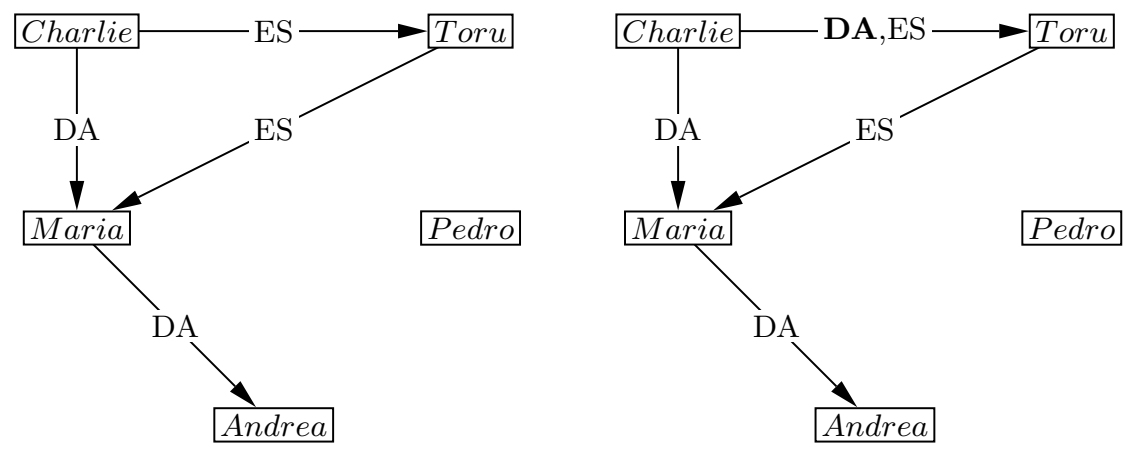

Fig. 2. The graph that shows for which agents the transmitted predicate holds in the state $s_{1}$ (left) $s_{2}$ (right) in Example 1. Changes with respect to state $s_{1}$ are in bold.

Given a set of agents $G \subseteq A g$ and a set of traits $T_{G} \subseteq \mathcal{T}$ we define the notions of weak sharing and strong sharing. 


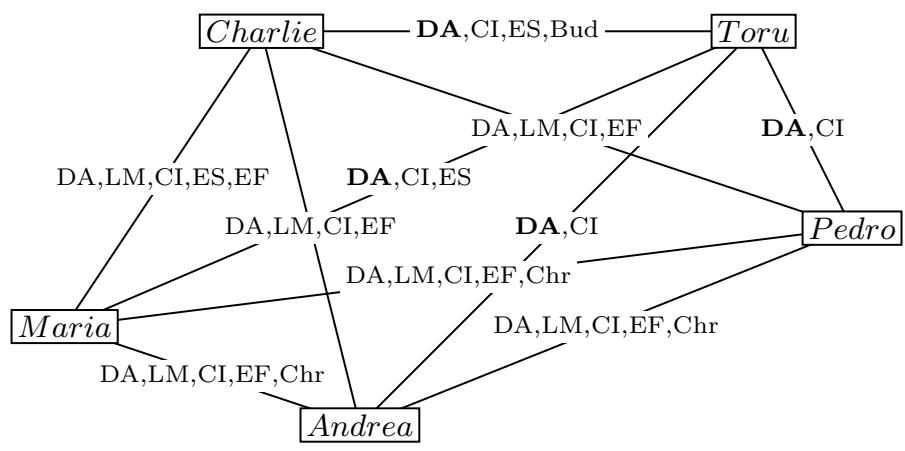

Fig. 3. The graph that shows for which agents the sharing predicate holds in the state $s_{2}$ in Example 1. Changes with respect to state $s_{1}$ are in bold.

Definition 4 (weak sharing). A set of traits $T_{G}$ is weakly shared by a set of agents $G$ in a state $s$ iff for each trait $\tau \in T_{G}$ there exists a pair of agents $a_{i}, a_{j} \in G, a_{i} \neq a_{j}$ that share $\tau$ in the state $s$.

Definition 5 (strong sharing). A set of traits $T_{G}$ is strongly shared by a set of agents $G$ in a state $s$ iff each trait $\tau \in T_{G}$ is shared by all pairs of agents $a_{i}, a_{j} \in G$ in $s$.

In other words, the set of traits is weakly (strongly) shared if it is a subset of the union (intersection) of traits shared by pairs of agents of $G$ in the state $s$.

Example 1 (continued). Let us consider the set of agents $G=\{$ Charlie, Toru, Maria, Andrea, Pedro\}. Analyzing the sharing predicate in the state $s_{1}$ (Figure 1) we can see that only the cappuccino_is_coffee trait is shared by each pair of agents in the state $s_{1}$, so $T_{G}=\{$ cappuccino_is_coffee $\}$ is strongly shared by $G$ in the state $s_{1}$. There are three traits that are shared by at least one pair of agents in the state $s_{1}$ : cappuccino_is_coffee, eating_with_sticks shared, e.g, by Toru and Charlie, and Dante_Alighieri_wrote_Divine_Comedy shared, e.g., by Charlie and Andrea. So, the set $T_{G}^{\prime}=\{$ Dante_Alighieri_wrote_Divine_Comedy, cappuccino_is_coffee, eating_with_sticks $\}$ and all non-empty subsets of this set are weakly shared by the set $G$ in the state $s_{1}$. Analogously, the set $T_{G}^{\prime \prime}=$ \{eating_with_sticks,Dante_Alighieri_wrote_Divine_Comedy, cappuccino_is_coffee\} is weakly shared by $G$ in the state $s_{3}$, and the set $T_{G}^{\prime \prime \prime}=\{$ cappuccino_is_coffee, Dante_Alighieri_wrote_Divine_Comedy $\}$ is strongly shared by the set $G$ in the state $s_{3}$.

Property 4. Strong sharing implies weak sharing.

Given a set of agents $G \subseteq A g$ such that $|G| \geq 2$, and a transmitted predicate we introduce the notion of culture of $G$.

Definition 6 (weak culture of a set of agents). A non-empty set of traits $T_{G} \subseteq \mathcal{T}$ is a weak culture of $G$ in a state $s$ iff 
- the set $T_{G}$ is weakly shared by $G$ in the state $s$,

- for each agent $a \in G$ in the state $s$ there exists a trait $\tau \in T_{G}$ such that has $(a, \tau, s)$.

From the assumption that traits are not innate, as we discussed, it follows that traits are acquired by agents, as represented by the transmitted predicate. Therefore, we can formulate the following axiom, telling that all traits in culture are transmitted.

Axiom 3 For each trait $\tau \in T_{G}$ there exists an agent $a \in A g$ that transmitted $\tau$ to another agent $a_{j} \in G$ before the state $s$, i.e. transmitted $\left(a, a_{j}, \tau, s\right)$.

From Definition 6 and Axiom 3 it follows that all the traits in the culture are transmitted, shared, and each agent has at least one trait from the culture. Please, note that since the traits are transmitted not necessarily within the set, the transmitted predicate does not imply sharing between the agents of $G$.

Definition 7 (strong culture of a set of agents). If $T_{G}$ in Definition 6 is also strongly shared in the state $s$ then it is a strong culture of the set of agents $G$ in the state $s$.

In the following if we refer to "a culture of a set of agents", we mean "a weak culture of a set of agents".

Example 1 (continued). Considering $G=\{$ Toru, Andrea $\}$ in the state $s_{3}, T_{G}=\{$ Dante_Alighieri_wrote_Divine_Comedy, cappuccino_is_coffee $\}$ is strongly shared by the set $G$ in the state $s_{3}$.

Although the Dante_Alighieri_wrote_Divine_Comedy trait has been transmitted both to Toru and Andrea from outside (from Charlie and Maria, respectively), it is strongly shared by the agents of $G$. Since in the state $s_{3}$ each agent in $G$ has the trait Dante_Alighieri_wrote_Divine_Comedy, $T_{G}^{\prime}=$ $\left\{\right.$ Dante_Alighieri_wrote_Divine_Comedy\} is a culture of $G$ in the state $s_{3}$. It is easy to see that $T_{G}^{\prime}$ is not a culture of $G$ in the states $s_{1}$ and $s_{2}$ because Toru does not have this trait in those states. $\oslash$

The following proposition outlines some restrictions on how culture can change between states, namely it shows that culture is monotonic.

Proposition 1 (monotonicity of culture). If a non-empty set of traits $T_{G}$ is a culture of a set of agents $G$ in a state $s_{v}$, then $T_{G}$ is a culture of $G$ also in any state $s$ after $s_{v}$.

In real world, the traits of a culture can be lost for two reasons: (1) agents can lose traits, (2) agents can die, move to another group, etc. As we stated in Axiom 1, in our model, agents do not lose traits. However, our model, and the proposition about monotonicity of culture support the case when agents disappear from the group.

Definition 8 (union culture of a group). A non-empty set of traits $T_{G}^{\text {union }}$ is the union culture of a set of agents $G$ in the state $s$ iff $T_{G}^{u n i o n}$ is the union of all cultures $T_{G}$ of $G$ in the state $s$. 
In other words, the union culture of a set of agents in some state is the union of all possible cultures of the set in this state. Since it is the union of all cultures, it is not possible to add any trait to $T_{G}^{u n i o n}$ and still obtain a culture of $G$. In the following, we refer to the union culture of a set of agents as "the culture of a set".

Definition 9 (evolution of culture). A sequence of sets of traits $\left\{T_{G}^{(1)}, \ldots\right.$, $\left.T_{G}^{(i)}\right\}$ is an evolution of culture of $G$ iff:

- exists a sequence of states $\left\{s_{1}, \ldots, s_{i}\right\}$, such that $T_{G}^{(k)}$ is a culture of $G$ in the state $s_{k}$ for all $k, 1 \leq k \leq i$,

- for each $k, 1 \leq k \leq i-1$ holds $i s \_a f t e r\left(s_{k+1}, s_{k}\right)$.

In other words, a sequence of sets of traits is an evolution of culture if each set of traits in the sequence is a culture of $G$ in some state and the states are ordered in the same way as the sets of traits. We denote evolution of culture as $\left\{T_{G}\right\}$.

\section{Related work and discussion}

Carley [7] considers culture as the distribution of information (ideas, beliefs, concepts, symbols, technical knowledge, etc.) across the population and proposes a model for knowledge transfer based on interactions. In that model, the probability of an interaction between two agents is based on the principle of homophily, i.e. the greater the amount of knowledge they share the more probable the interaction is. During an interaction, agents exchange facts, so after the interaction one of the agents might know more than before the interaction. The knowledge transfer in these settings can be seen as a particular kind of culture spread. This work is further extended in the Construct project [28,29]. For instance, one of the recent applications of Construct studies the effects of different methods of information diffusion on spreading beliefs and knowledge about illegal tax schemes in different American cities [30]. With respect to the definition of culture we propose in this paper, that model of information diffusion is complementary, because it models transmission of elements of culture (e.g., beliefs, knowledge) in a society.

Axelrod [1] considers culture as a list of features or dimensions. Each feature represents an individual attribute that is subject to social influence and can have different values called traits. Two individuals have the same culture if they have the same traits for all features. Similarly to the work by Carley, feature of an agent can change its value during an interaction and the probability of interaction is based on the homophily.

The notion of trait we use in our formalism is similar to the notion of feature used by Axelrod, specifically, each feature can take value from a set of specific traits. Traits in our formalism also includes ideas, beliefs and technical knowledge used as culture elements by Carley. Both theories by Carley and by Axelrod are based on the assumption that culture changes as a result of an interaction. Thus, in our terms, interaction in that sense can be considered as a particular kind of 
transmission: there are two agents participating, it takes place in some specific state and it leads to the appearance of some cultural element in one of the agents.

Epstein and Axtell [8] study the emergence of the group rules from local ones defined at an agent's level in an artificial society of simple agents living and consuming sugar in an artificial environment called "Sugarscape". The authors consider a culture of the society as a string of binary cultural attributes and model cultural transmission both on horizontal (between agents) and vertical (through generations) levels using simple rules. However, they do not provide any formal definition of culture since the main focus of the book is on the emergence of group rules from the local ones.

According to O'Reilly [13], the culture of an organization is considered as strong if wide consensus exists about the content and participants believe in the importance of the content. They also formulate this as a [not necessarily big] set of values that are widely shared and strongly held. This is similar to the notion of strong culture, i.e. culture shared by all pairs of agents in a group, we consider in our formalism.

Balzer and Tuomela [31] study social practices and the dynamics of their maintenance in groups. They define social practices as recurrent collective activities based on collective intentions. The paper focuses on informal, non-normative practices, such as playing soccer on Sundays, going to sauna on Saturday afternoon, shaking hands, sharing a ride to work. They also note that the maintenance (change, preservation, renewal) depend on the success of a practice. The main contribution of the paper is a mathematical model for the description of social practices and their maintenance in groups.

Our model of culture is not limited to social practices. Moreover, it allows for inclusion of normative practices as well. However, as a consequence, the model of Balzer and Tuomela allows for a richer description of informal social practices. For instance, our model does not permit expressing intentions, but allows operating on manifestations of activities without going into details of underlying intentions. While authors show that success of a social practice is important for its adoption, for our model it is irrelevant whether a trait is successful in some sense. Our model just captures the fact that the trait is a part of culture, no matter how it occurred. The model presented by Balzer and Tuomela is defined for groups and then goes to the individual level, thereby implementing top-down approach. In our model of culture, we start from a set of traits of an individual, consider transmission as an important means of spreading culture, and then go to the culture of a group. Thus, we implement bottom-up approach. Balzer and Tuomela, while requiring sharing of a social practice within a group, and noting the importance of transmission for spreading practice, include transmission into the model only to a certain extent, namely, considering imitation as an example of transmission. Our model of culture allows for different types of transmission as long as there is a predicate that helps to distinguish occurred transmissions.

Hofstede [9] treats culture as "[...] the collective programming of the mind that distinguishes the members of one group or category of people from another", proposes a model of culture and applies it for studying and comparing cultures 
of IBM workers in more than 50 countries. The model includes the following five independent dimensions of national culture differences: power distance, which is related to the different solutions to the basic problem of human inequality; unvertainty avoidance, which is related to the level of stress in a society in the face of an unknown future; individualism versus collectivism, which is related to the integration of individuals into primary groups; masculinity versus femininity, which is related to the division of emotional roles between men and women; and long-term versus short-term orientation, which is related to the choice of focus for people's efforts: the future or the present. Values in Hofstede's terms refer to "a broad tendency to prefer certain states over others" and are similar to attitudes and beliefs, which are just particular kind of traits in our formalism. Dimensions, similarly to Axelrod's features, take values from the set of traits. Thus, comparing with our work, the model developed by Hofstede has a different focus - it aims at comparing cultures of groups of people over several pre-defined dimensions of values, while our model supports comparison over arbitrary sets of traits. The dimensions in Hofstede's model are meant to be independent, while our formalism does not address the issue of dependency of traits, so they can be dependent on each other. In this line of thoughts, an interesting application of our model could be comparison of dependency of traits across groups, i.e. if presence of a trait or traits leads to the presence of another trait(s) for one group and to the presence of third trait(s) for another group.

The definition of culture presented here allows for representation and comparison of different cultures. However, in order to compare traits, one first needs to identify the traits of individuals. On the one hand, deducing traits from manifested behaviors of agents is not a trivial task in general. On the other hand, in specific domains this might be much easier, consider, for instance, deducing traits of users from logs of a web service, website, or an application. For instance, it would be possible to see that a group of users of a text editor always turn off the autocorrect feature and turn it off automatically in new versions of the editor prepared for this group. Taking the issue of the observability of traits into account, we see social software and Web 2.0 systems as one of the potential application domains for our model.

\section{Conclusion}

In this paper we have presented a formal definition of culture of a set of agents. This definition addresses existing gaps in AI literature that deals with issues of sociality, cooperation, and negotiation, but remains oblivious to the notion of culture. The formalism presented in this paper is a part of ongoing research and we are currently working on measures for characterizing community culture and on studying of evolution of culture in Web 2.0 communities. 


\section{Acknowledgments}

This work has been supported by the European Union FP7 projects COMPAS and LiquidPublication, grants no. 215175 and 213360. We would like to thank Stefano Borgo for suggestions he provided for improving the paper.

\section{References}

1. Axelrod, R.: The dissemination of culture: A model with local convergence and global polarization. The Journal of Conflict Resolution 41(2) (1997) 203-226

2. Castelfranchi, C.: Modelling social action for ai agents. Artificial Intelligence 103(1-2) (1998) 157-182

3. Ossowski, S.: Co-ordination in Artificial Agent Societies, Social Structure and Its Implications for Autonomous Problem-Solving Agents. Volume 1535 of Lecture Notes in Computer Science. Springer (1999)

4. Shoham, Y., Tennenholtz, M.: On social laws for artificial agent societies: off-line design. Artif. Intell. 73(1-2) (1995) 231-252

5. Shoham, Y., Tennenholtz, M.: On the emergence of social conventions: modeling, analysis, and simulations. Artificial Intelligence 94(1-2) (1997) 139-166

6. Masolo, C., Vieu, L., Bottazzi, E., Catenacci, C., Ferrario, R., Gangemi, A., Guarino, N.: Social roles and their descriptions. In: Principles of Knowledge Representation and Reasoning (KR2004). (2004)

7. Carley, K.: A theory of group stability. American Sociological Review 56(3) (1991)

8. Epstein, J.M., Axtell, R.L.: Growing Artificial Societies: Social Science from the Bottom Up. The MIT Press (1996)

9. Hofstede, D.G.: Culture's Consequences: Comparing Values, Behaviors, Institutions and Organizations Across Nations. 2nd edn. Sage Publications, Inc (April 2001)

10. Mulder, M.B., Nunn, C.L., Towner, M.C.: Cultural macroevolution and the transmission of traits. Evolutionary Anthropology 15(2) (2006) 52-64

11. Mead: Cooperation and Competition Among Primitive Peoples. Transaction Publishers (2002)

12. Brumann, C.: Writing for culture: Why a successful concept should not be discarded [and comments and reply]. Current Anthropology 40 (1999)

13. O'Reilly, C.: Corporations, culture, and commitment: Motivation and social control in organizations. California Management Review 31(4) (1989) 9-25

14. Mead, M., Métraux, R., eds.: The Study of Culture at a Distance. University of Chicago Press (1953)

15. McFate, M.: The military utility of understanding adversary culture. Joint Force Quarterly 38 (2005)

16. Bozeman, A.B.: Strategic Intelligence and Statecraft: Selected Essays. Washington, DC: Brassey's (1992)

17. Boyd, R., Richerson, P.J.: Culture and the Evolutionary Process. University of Chicago Press (1988)

18. Kroeber, A.L., Kluckhohn, C.: Culture: A critical review of concepts and definitions. Papers of the Peabody Museum 47 (1952) 643-656

19. Harrison, R.J., Carroll, G.R.: Culture and Demography in Organizations. Princeton University Press (2006) 
20. Schein, E.H.: Organizational Culture and Leadership (Jossey-Bass Psychology Series). 2 edn. Jossey-Bass (1996)

21. Bailey, G., Peoples, J.: Introduction to Cultural Anthropology. Wadsworth Publishing (1998)

22. Keesing, R.M.: Cultural anthropology: A contemporary perspective. Holt, Rinehart and Winston (1981)

23. Tylor, E.B.: Primitive culture. London: J. Murray (1871)

24. Linton, R.: The study of man: An introduction. New York: D. Appleton-Century (1936)

25. Harris, M.: Culture, people, nature: An introduction to general anthropology. New York: Thomas Y. Crowell (1975)

26. Ferber, J.: Multi-Agent Systems: An Introduction to Distributed Artificial Intelligence. Addison-Wesley Professional (1999)

27. Sengers, P.: Anti-Boxology: Agent Design in Cultural Context. PhD thesis, Carnegie Mellon University (1998)

28. Hirshman, B.R., Carley, K.M., Kowalchuck, M.J.: Specifying agents in construct. Technical Report CMU-ISRI-07-107, Carnegie Mellon University (2007)

29. Hirshman, B.R., Carley, K.M., Kowalchuck, M.J.: Loading networks in construct. Technical Report CMU-ISRI-07-116, Carnegie Mellon University (2007)

30. Hirshman, B.R., Birukou, A., Martin, M.K., Bigrigg, M.W., Carley, K.M.: The impact of educational interventions on real \& stylized cities. Technical Report CMU-ISR-08-114, Carnegie Mellon University (2008)

31. Balzer, W., Tuomela, R.: Collective intentions and the maintenance of social practices. Autonomous Agents and Multi-Agent Systems 6(1) (2003) 7-33 